\title{
IN-PLANE/TILTED \\ MAGNETIC-FIELD-DEPENDENT CONDUCTANCE OF 2D ELECTRON SYSTEMS IN ASYMMETRIC DOUBLE QUANTUM WELLS
}

\author{
L. Smrŏka, P. Vašek, T. Jungwirth, O.N. Makarovskii*, M. Cukr \\ Institute of Physics, Academy of Science of the Czech Republic \\ Cukrovarnická 10, 16200 Prague 6, Czech Republic
}

AND L. JANSEN

Grenoble High Magnetic Field Laboratory, B.P. 166, 38042 Grenoble Cedex 9, France

The resistance in an asymmetric double-well structure was measured as a function of magnetic fields oriented almost parallel to the plane of the electron layer. It was shown that the shape of the magnetoresistance curves is close to the in-plane magnetic-field-dependent density of states which we obtained by self-consistent numerical calculation. The novel feature is the negative magnetoresistance observed at low magnetic fields.

PACS numbers: $72.20 . \mathrm{My}, 73.40 . \mathrm{Kp}_{\mathrm{p}}$

Two-dimensional electron gas (2DEG) confined in semiconductor double quantum wells and/or wide single quantum wells has been extensively studied over the past several years. It was found that a single-layer/bilayer transition of the electron system can be caused by application of a magnetic field $B_{\|}$parallel to the growth plane of structures and that such transition has a strong influence on the electron transport properties of GaAs/AlGaAs double wells [1, 2] and wide single quantum wells [3].

The essence of physics of a single-layer/bilayer transition is captured by a simple tight-binding model of tunnelling between two wells. The model predicts the in-plane-field-induced shifts of the Fermi circles of two electron layers in the opposite directions in the wave vector space and, further, their anticrossing, resulting in new "peanut" and "lens" shapes of the Fermi lines. At low fields, the lines become close to two concentric circles; when the magnetic field is increased the lens is emptied followed by splitting of the peanut into two parts. The changes of topology of the Fermi lines are accompanied by variation of the magnetic-field

*On leave from Institute of Radiophysics and Electronics, NAS Ukr., Kharkov, Ukraine. 
dependence of the density of states (DOS). Depopulation of the lens gives rise to a step while splitting of the peanut results in a logarithmic singularity in the DOS.

Here we report the study of an asymmetric GaAs/AlGaAs double well. The structure was prepared by MBE technique: a thin barrier, formed by six monolayers of $\mathrm{AlGaAs}$, was grown in the GaAs layer of a standard heterostructure at the distance $\approx 6 \mathrm{~nm}$ from the interface. In this way, the original triangular well was divided into a distorted rectangular well and a secondary triangular well. It follows from the measurement of the Shubnikov-de Haas oscillations in perpendicular magnetic fields at $4.2 \mathrm{~K}$ that only the subband corresponding to symmetric (bonding) combination of states of individual wells is occupied. This measurement and the low-field measurement of the Hall resistance determine the concentration $N_{s}$ and the mobility $\mu$ of the 2DEG: $N_{s}=2.8 \times 10^{-11} \mathrm{~cm}^{-2}, \mu=3.4 \times 10^{5} \mathrm{~cm}^{2} /(\mathrm{V} \mathrm{s})$.

The strong coupling of wells, resulting in an occupation of a single subband, was confirmed by a self-consistent calculation of the electron energy spectra. The coupled Schrödinger and Poisson equations were solved numerically, together with the formula describing exchange-correlation effect in the local-density-functional approximation [4]. According to the results of this calculation for the case $B_{\|}=0$, the antisymmetric level lies slightly above the Fermi energy and the barrier between wells roughly halves the electron charge in our system.

The calculation, extended to nonzero in-plane magnetic fields $B_{\|}$, describes quantitatively the single-layer/bilayer transition. Results of calculations presented in Fig. 1 illustrate the gradual progress of the transition as seen in the $k$-space. The transition is manifested by distortion of the Fermi lines calculated for several

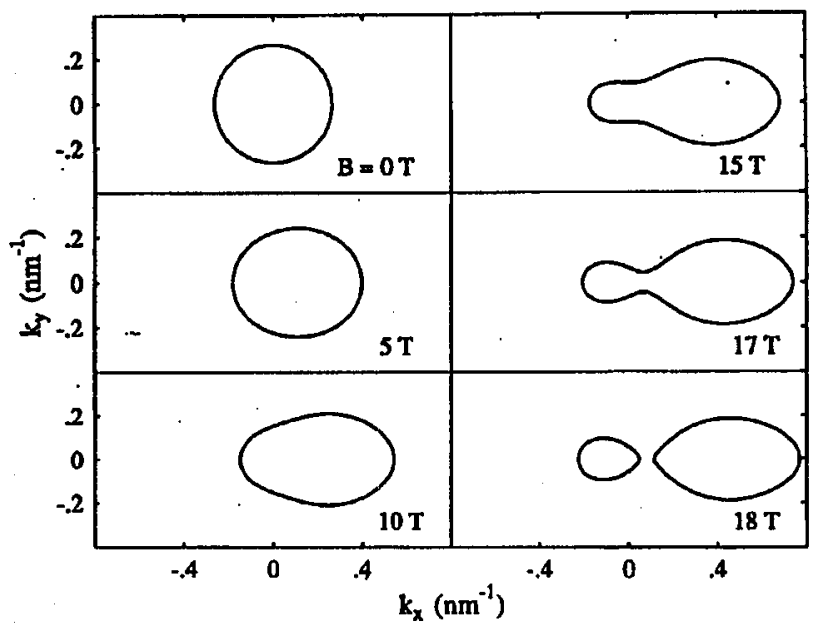

Fig. 1. Fermi lines at different parallel magnetic fields, calculated self-consistently within the local density approximation.

$B_{\|}$ranging from 0 to $18 \mathrm{~T}$, the splitting of the asymmetric peanut occurs at $B_{\|}^{\mathrm{c}} \approx 17.5 \mathrm{~T}$. To translate this $k$-space picture to terms of the charge redistribution in the real space, we start with discussion of two separated Fermi lines at $B_{\|}>B_{\|}^{\mathrm{c}}$. 
They originate in the Fermi lines of two independent electron layers in two wells which do not cross due to the large field-induced shift of their centers and are only slightly modified by the coupling of the wells. At a field just below the splitting field $B_{\|}^{\mathrm{c}}$, two Fermi lines are connected by a narrow neck, which originates from the tunneling-induced anticrossing of Fermi lines belonging to individual wells. For $k$ vectors at the neck, the bound states of uncoupled wells are combined into bonding states, while at the rest of the peanut line the electrons stay predominantly in one of the wells. At lower magnetic fields, the central part of the Fermi line, common to both wells, becomes larger and, finally, at $B_{\|}=0$, all states at the Fermi line are formed by a symmetric combination of individual quantum well states.

The magnetoresistance curves measured as functions of magnetic fields parallel to or slightly tilted from the 2D-layer plane are shown in Fig. 2 for the total magnetic field $B$ ranging from 0 to $23 \mathrm{~T}$ and for the temperature as low as $0.44 \mathrm{~K}$. In a strictly in-plane configuration and with the measuring current

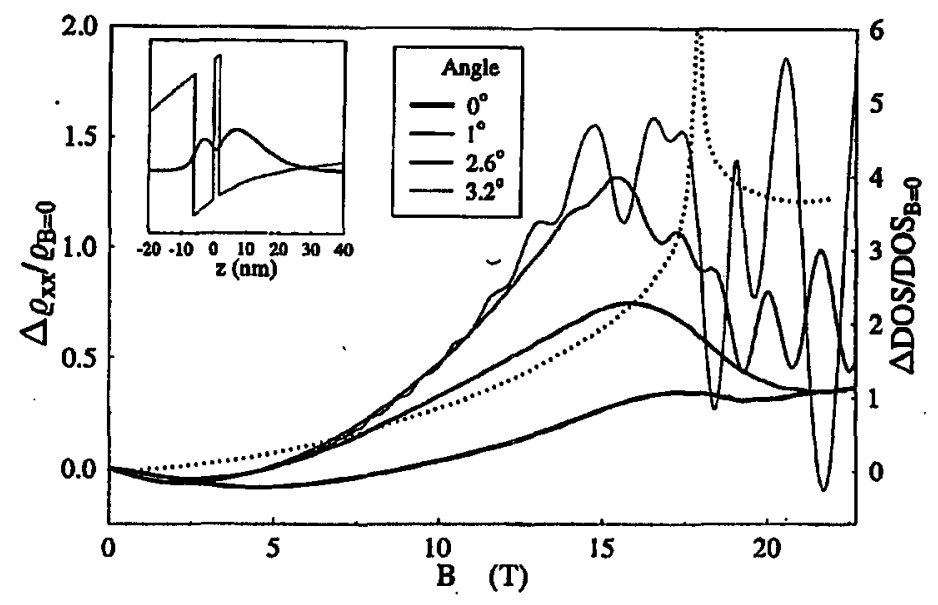

Fig. 2. Magnetic field dependence of the magnetoresistance measured at small tilt angles. The dotted line denotes the DOS calculated for the in-plane field configuration. The inset shows the charge distribution in the quantum wells at $B=0$.

parallel to $B_{\|}$, the experimental dependence of the magnetoresistance follows the gross features of the field dependent DOS, also shown in Fig. 2. It rises at intermediate magnetic fields and reaches maximum close to the point where the DOS exhibits the logarithmic singularity. This is in agreement with previous studies on double quantum wells and wide single quantum wells. The novel feature here is a negative magnetoresistance observed for fields below $5 \mathrm{~T}$. We attribute it to the magnetic-field-induced progressive separation of electrons to different wells, allowing the current to flow predominantly in the secondary triangular well, more remount from the ionized donors. At higher fields, the increase in DOS takes over this effect.

In tilted magnetic fields, the strong increase in resistance with the tilt angle, i.e with the growing perpendicular component of the field, $B_{\perp}$, is observed for 
a given $B_{\|}$. This behaviour is in agreement with the previous measurements on standard GaAS/AlGaAs heterostructures with approximately triangular quantum wells $[5,6]$ and can be understood within the framework of a standard magnetotransport theory [7]. According to this theory the magnetoresistance of the system with a closed Fermi line of a noncircular shape and an anisotropic relaxation time is positive and saturates at high $B_{\perp}$. In our case, $B_{\|}$determines the shape of the Fermi line and the anisotropy of the relaxation time $\tau$, while $B_{\perp}$ drives electrons along the Fermi line with the cyclotron frequency $\omega_{\mathrm{c}}=|e| B_{\perp} / m_{\mathrm{c}}$. The cyclotron effective mass $m_{c}$ is related to the density of states $g$ by $g=m_{c} / \pi \hbar^{2}$ and, therefore, shares its in-plane field dependence. The product of $\omega_{\mathrm{c}} \tau$ is decisive for the $B_{\perp}$-dependence of the magnetoresistance, which saturates for $\omega_{\mathrm{c}} \tau \gg 0$. The higher saturation fields of the magnetoresistance near the critical field $B_{\|}^{c}$ can be explained by the variation of $m_{\mathrm{c}}$ with $B_{\|}$. The cyclotron mass reaches the maximum values at $B_{\|}^{\mathrm{C}}$ and, therefore, the condition $\omega_{\mathrm{c}} \tau \gg 0$ is satisfied for higher $B_{\perp}$. As for the $\tau$, we expect that its anisotropy strongly grows with $B_{\|}$due to the separation of electrons into individual wells. Above the $B_{\|}^{\mathrm{c}}$, two cyclotron masses and two relaxation times are defined for two Fermi lines.

For $\omega_{\mathrm{c}} \tau \gg 0$ the Shubnikov-de Haas oscillations have the amplitude determined by $k_{\mathrm{B}} T / \hbar \omega_{\mathrm{c}}$. From their temperature damping, the $B_{\|}$-dependent cyclotron effective mass can be determined. While the data do not allow for a quantitative determination of $m_{c}$, it is obvious that $m_{\mathrm{c}}$ grows with $B_{\|}$and culminates at $B_{\|} \approx B_{\|}^{c}$. Above $B_{\|}^{c}$, two new smaller effective masses are identified.

The magnetotransport measurements were done in Grenoble High Magnetic Field Laboratory.

\section{References}

[1] S.K. Lyo, Phys. Rev. B 50, 4965 (1994).

[2] J.A. Simmons, N.E. Harff, J.F. Klem, Phys. Rev. B 51, 11156 (1995).

[3] T. Jungwirth, T.S. Lay, L. Smrčka, M. Shayegan, Phys. Rev. B 56, (1997), in press.

[4] L. Hedin, B.I. Lundquist, J. Phys. C 4, 2064 (1971); F. Stern, S. Das Sarma, Phys. Rev. B 30, 840 (1984).

[5] D.R. Leadley, R.J. Nicholas, J.J. Harris, C.T. Foxon, in: Proc. 20lh Int. Conf. on Physics of Semiconductors, Eds. E.M. Anastassakis, J.D. Joannopoulos, World Scientific, Singapore 1990, p. 1609.

[6] P. Středa, P. Vašek, M. Cukr, Phys. Rev. B 51, 11144 (1995).

[7] A.B. Pippard, Magnetoresistance in Metals, Cambridge University Press, Cambridge 1989, p. 24. 